\title{
Effects of renormalizing the chiral SU(2) quark-meson model
}

\author{
Andreas Zacchi ${ }^{*}$ and Jürgen Schaffner-Bielich ${ }^{\dagger}$ \\ Institut für Theoretische Physik, Goethe Universität Frankfurt, \\ Max von Laue Strasse 1, D-60438 Frankfurt, Germany
}

(Received 14 December 2017; published 12 April 2018)

\begin{abstract}
We investigate the restoration of chiral symmetry at finite temperature in the $\mathrm{SU}(2)$ quark-meson model, where the mean field approximation is compared to the renormalized version for quarks and mesons. In a combined approach at finite temperature, all the renormalized versions show a crossover transition. The inclusion of different renormalization scales leave the order parameter and the mass spectra nearly untouched but strongly influence the thermodynamics at low temperatures and around the phase transition. We find unphysical results for the renormalized version of mesons and the combined one.
\end{abstract}

DOI: 10.1103/PhysRevD.97.074011

\section{INTRODUCTION}

Since QCD is non-perturbative in the low energy regime, effective theories and models based on the QCD Lagrangian and its properties have to be utilized [1-4]. The QCD Lagrangian possesses an exact color and flavor symmetry for $N_{f}$ massless quark flavours [5-11], and chiral symmetry controls the hadronic interactions in the low energy regime $[12,13]$. At high temperatures or densities chiral symmetry is expected to be restored [14,15]. In general, the interaction can be modeled by the exchange of scalar, pseudoscalar, and vector mesons [16]. If one adopts the linear sigma model $[17,18]$ for quark interactions, it is referred to as the chiral quark-meson model [10,19-22], which is well studied [23-30]. Its advantage in comparison to other chiral effective models like the Nambu-Jona-Lasinio (NJL) model [31-34] lies in its renormalizability. Renormalizability takes into account the contribution of vacuum fluctuations [23,35-37]. Works which included the vacuum term by using the renormalization group flow equations focused in particular on the neighborhood of critical points [2,21,38,39].

In this article, we study quarks, by using a chiral $\mathrm{SU}(2)$ quark-meson model within the path integral formalism, and mesons, which are examined within the 2PI formalism, within a combined approach. We investigate this approach also in the mean field approximation and consider the vacuum term contribution, which depends on a

\footnotetext{
zacchi@astro.uni-frankfurt.de

schaffner@astro.uni-frankfurt.de
}

Published by the American Physical Society under the terms of the Creative Commons Attribution 4.0 International license. Further distribution of this work must maintain attribution to the author(s) and the published article's title, journal citation, and DOI. Funded by SCOAP. renormalization scale resulting from the inclusion of the meson fields.

Besides the order parameter and the masses of the sigma and the pion, we study thermodynamical quantities. In all cases studied, the masses of the pion and the sigma meson start to be degenerate around the phase transition, which is defined by the order parameter. The impact of the meson contribution on the order parameter and mass is comparatively small, whereas thermodynamic quantities are strongly influenced. At low temperatures, the impact of the mesonic contribution is substantial within the combined approach. In our approach, we vary the mass of the sigma meson in the range $500 \leq m_{\sigma}^{\mathrm{vac}} \leq 900 \mathrm{MeV}$. For the standard value of $m_{\sigma}=550 \mathrm{MeV}$ we find a smooth chiral crossover phase transition around the critical temperature $T_{c} \simeq 155 \mathrm{MeV}[2,29]$. We compare our studies for the quark fields with works from Refs. [23-25] and for the mesonic fields with works from Refs. [26-29]. In the combined approach, we compare our results with the work from Ref. [2], in which the authors derive an effective action for the meson fields and linearize it around the ground state.

We find that the renormalization scale cancels when considering the SU(2) quark-meson model for the quark fields, and the inclusion of the vacuum term shifts the phase transition to larger temperatures. The combined model is dependent on the renormalization scales. Hence, a combined model for quarks and mesons is only acceptable in the mean field approximation.

\section{GENERAL CONSIDERATIONS}

Before going into more details, we briefly sketch a general consideration to show that the approach used is thermodynamically consistent. A general ansatz for the effective action $\Gamma[\phi, G, Q]$ according to $[2,26,40,41]$ is 


$$
\begin{aligned}
\Gamma[\phi, G, Q]= & I[\phi]-\frac{1}{2} \operatorname{Tr}\left(\ln G^{-1}\right)-\frac{1}{2} \operatorname{Tr}\left(D^{-1} G-1\right) \\
& +\operatorname{Tr}\left(\ln Q^{-1}\right)+\operatorname{Tr}\left(S^{-1} Q-1\right) \\
& +\Gamma_{2}[\phi, G, Q]
\end{aligned}
$$

where $\phi$ represents the fields involved, and $I[\phi]$ is the classical action or the tree-level potential. $G$ is the full propagator and $D^{-1}$ the inverse tree-level propagator for the mesons. $Q$ is the full propagator and $S^{-1}$ the inverse treelevel propagator for the quarks. $\Gamma_{2}[\phi, G, Q]$ is the contribution from the two-particle irreducible diagrams, which in our case only depends on the fields and the full propagator of the mesons, i.e. $\Gamma_{2}[\phi, G]$; see also Fig. 2.

In the absence of sources, the stationary conditions determine the vacuum expectation values of $\phi$. They read

$$
\begin{gathered}
\frac{\delta \Gamma[\phi, G, Q]}{\delta \phi}=\frac{\delta I[\phi]}{\delta \phi}-\frac{1}{2} \operatorname{Tr}\left(\frac{\delta D^{-1}}{\delta \phi} G\right) \\
+\operatorname{Tr}\left(\frac{\delta S^{-1}}{\delta \phi} Q\right)+\frac{\delta \Gamma_{2}[\phi, G]}{\delta \phi}=0, \\
\frac{\delta \Gamma[\phi, G, Q]}{\delta G}=-\frac{1}{2} D^{-1}+\frac{1}{2} G^{-1}+\frac{\delta \Gamma_{2}[\phi, G]}{\delta G}=0, \\
\frac{\delta \Gamma[\phi, G, Q]}{\delta Q}=-Q^{-1}+S^{-1}=0 .
\end{gathered}
$$

Since no contribution from $\Gamma_{2}[\phi, G]$ to the stationary conditions occurs for the quark propagator $Q$, no diagrams containing a quark propagator within a meson loop appear within our approach. Hence, it is justified to evaluate the potentials independently, and the respective gap equations in the combined approach are consequently additive.

In the following, we briefly sketch the derivation of the individual approaches to finally combine them.

\section{QUARK-QUARK INTERACTION}

A Lagrangian with $N_{f}=2$ respecting quark fields may be written as $[19,20,26,27]$

$$
\begin{aligned}
\mathcal{L}= & \mathcal{L}_{q}+\mathcal{L}_{m}-U(\sigma, \vec{\pi}) \\
= & \bar{\Psi}\left(i \not \partial-g\left(\sigma+i \gamma_{5} \vec{\tau} \cdot \vec{\pi}\right)\right) \Psi \\
& +\frac{1}{2}\left(\partial_{\mu} \sigma \partial^{\mu} \sigma+\partial_{\mu} \vec{\pi} \partial^{\mu} \vec{\pi}\right)-U(\sigma, \vec{\pi}),
\end{aligned}
$$

where $g=m_{q, \mathrm{vac}} / f_{\pi}$ is a Yukawa type coupling to the quark spinors $\Psi$. Here, $m_{q \text {,vac }}$ is the constituent quark mass chosen to be $300 \mathrm{MeV}$ and $f_{\pi}=92.4 \mathrm{MeV}$ the pion decay constant [11]. $U(\sigma, \vec{\pi})$ is the tree-level potential and is given as

$$
U(\sigma, \vec{\pi})=\frac{\lambda}{4}\left(\left(\sigma^{2}+\vec{\pi}^{2}\right)-v^{2}\right)^{2}-H \sigma
$$

with the coupling $\lambda$ and the mass term $m=-\lambda v^{2}$. The term $H$ breaks chiral symmetry explicitly and is therefore responsible for the nonvanishing mass of the pion $[19,20,42-44]$. The grand canonical potential is commonly derived with the path integral formalism [2,39,45-48] and reads

$$
\begin{gathered}
\Omega_{\bar{q} q}=U(\sigma, \vec{\pi})+\Omega_{\bar{q} q}^{\mathrm{th}}+\Omega_{\bar{q} q}^{\mathrm{vac}} \\
=\frac{\lambda}{4}(\sigma+\vec{\pi})^{4}+\frac{m^{2}}{2}(\sigma+\vec{\pi})^{2}+\frac{\lambda v^{4}}{4}-H \sigma \\
-N_{f} N_{c} T \int_{0}^{\infty} \frac{d k^{3}}{\left(2 \pi^{3}\right)}\left[\ln \left(1+e^{-\beta\left(E_{k} \pm \mu_{f}\right)}\right)\right] \\
-N_{f} N_{c} T \int_{0}^{\infty} \frac{d k^{3}}{\left(2 \pi^{3}\right)}\left(\frac{E}{T}\right) .
\end{gathered}
$$

Here, $N_{c}=3$, the single particle energy

$$
E_{k}=\sqrt{k^{2}+\tilde{m}_{f}^{2}} \quad \text { with } \quad \tilde{m}_{f}=g \sqrt{\sigma^{2}+\vec{\pi}^{2}}
$$

as the effective mass, and $\mu_{f}$, as the flavor dependent quark chemical potential, have been introduced. The term of line (12) represents the contribution due to vacuum fluctuations. Solutions are then obtained by solving

$\frac{\partial \Omega_{\bar{q} q}}{\partial \sigma} \stackrel{!}{=} 0, \quad \frac{\partial^{2} \Omega_{\bar{q} q}}{\partial \sigma^{2}}=m_{\sigma}, \quad$ and $\quad \frac{\partial^{2} \Omega_{\bar{q} q}}{\partial \vec{\pi}^{2}}=m_{\vec{\pi}}$

also known as gap equations. The derivatives read

$$
\begin{aligned}
\left.\frac{\partial \Omega_{\bar{q} q}^{\mathrm{th}}}{\partial \sigma}\right|_{T=\mu=0} & =\left.\frac{\partial U(\sigma, \vec{\pi})}{\partial \sigma}\right|_{\vec{\pi}=0} \\
& =\lambda\left(\sigma^{2}-v^{2}\right) \sigma-H \\
\left.\frac{\partial \Omega_{\bar{q} q}^{\mathrm{th}}}{\partial \vec{\pi}}\right|_{T=\mu=0} & =\left.\frac{\partial U(\sigma, \vec{\pi})}{\partial \vec{\pi}}\right|_{\vec{\pi}=0} \\
& =\lambda\left(\vec{\pi}^{2}+\sigma^{2}-v^{2}\right) \vec{\pi} .
\end{aligned}
$$

Equation (15) gives the minimum of the potential, which is identified as the ground state of a particle. Note that Eq. (16), i.e. the first derivative of $\Omega_{\bar{q} q}^{\text {th }}$ with respect to $\vec{\pi}$, yields zero. This is reasonable since the $\vec{\pi}$ does not condensate due to its negative parity. The vacuum expectation value of the sigma field is the pion decay constant, $f_{\pi}$, which is an input parameter. The second derivatives read 


$$
\begin{aligned}
\left.\frac{\partial^{2} \Omega_{\bar{q} q}^{\mathrm{th}}}{\partial \sigma^{2}}\right|_{T=\mu=0} & =\left.\frac{\partial U^{2}(\sigma, \vec{\pi})}{\partial \sigma^{2}}\right|_{\vec{\pi}=0} \\
& =\lambda\left(3 \sigma^{2}-v^{2}\right)=m_{\sigma, \mathrm{vac}}^{2} \\
\left.\frac{\partial^{2} \Omega_{\bar{q} q}^{\mathrm{th}}}{\partial \vec{\pi}^{2}}\right|_{T=\mu=0} & =\left.\frac{\partial U^{2}(\sigma, \vec{\pi})}{\partial \vec{\pi}^{2}}\right|_{\vec{\pi}=0}=0 \\
& =\lambda\left(\sigma^{2}-v^{2}\right)=m_{\pi, \mathrm{vac} .}^{2}
\end{aligned}
$$

and yield the respective mass spectrum of the particles, which we used as input parameters to determine $\lambda, v^{2}$, and $H$; see also [30]. Using Eqs. (15), (17), and (18), one can extract the values for

$$
\begin{gathered}
\lambda=\frac{m_{\sigma}^{2}-m_{\pi}^{2}}{2 f_{\pi}^{2}}, \\
v^{2}=f_{\pi}^{2}-\frac{m_{\pi}^{2}}{\lambda} \quad \text { or } \quad v^{2}=3 f_{\pi}^{2}-\frac{m_{\sigma}^{2}}{\lambda}, \\
H=m_{\pi}^{2} f_{\pi}
\end{gathered}
$$

in the vacuum.

The vacuum parameters can be found in Table II.

\section{A. Regularization for the quark fields}

Taking into account vacuum fluctuations needs regularization schemes $[2,26,27,35]$. To regularize the divergencies, we use dimensional regularization.

The vacuum term in Eq. (9) [Eq. (12)] is, to lowest order, just the one-loop effective potential at zero temperature and reads in $d=3-2 \epsilon$ dimensions, where $\lim \epsilon \rightarrow 0$, regularized [35]

$\Omega_{\bar{q} q}^{\mathrm{vac}}=\frac{N_{c} N_{f}}{16 \pi^{2}} \tilde{m}_{f}^{4}\left[\frac{1}{\epsilon}-\frac{1}{2}\left[-3+2 \gamma+4 \ln \left(\frac{\tilde{m}_{f}}{2 \sqrt{\pi} \Lambda}\right)\right]\right.$.

Here, $\gamma$ is the Euler-Mascheroni constant and $\Lambda$ an arbitrary renormalization scale parameter. To renormalize the thermodynamic potential, an appropriate counterterm $\delta \mathcal{L}$ needs to be introduced to the Lagrangian [35]. The minimal substraction $(\overline{\mathrm{MS}})$ scheme allows for

$$
\delta \mathcal{L}=\frac{N_{c} N_{f}}{16 \pi^{2}} \tilde{m}_{f}^{4}\left[\frac{1}{\epsilon}-\frac{1}{2}[-3+2 \gamma-4 \ln (2 \sqrt{\pi})]\right],
$$

and the renormalized vacuum contribution becomes

$$
\Omega_{\bar{q} q}^{\mathrm{vac}} \rightarrow \Omega_{\bar{q} q}^{d r}=-\frac{N_{c} N_{f}}{8 \pi^{2}} \tilde{m}_{f}^{4} \ln \left(\frac{\tilde{m}_{f}}{\Lambda}\right)
$$

The vacuum contributions to the gap equations, Eqs. (14), due to Eq. (24), are

$$
\begin{gathered}
\frac{\partial \Omega_{\bar{q} q}^{d r}}{\partial \sigma}=-\frac{N_{c} N_{f} g^{4} \sigma^{3}}{8 \pi^{2}}\left[1+4 \ln \left(\frac{\sigma}{f_{\pi}}\right)\right], \\
\frac{\partial^{2} \Omega_{\bar{q} q}^{d r}}{\partial \sigma^{2}}=-\frac{N_{c} N_{f} g^{4} \sigma^{2}}{8 \pi^{2}}\left[7+12 \ln \left(\frac{\sigma}{f_{\pi}}\right)\right], \\
\frac{\partial^{2} \Omega_{\bar{q} q}^{d r}}{\partial \vec{\pi}^{2}}=-\frac{N_{c} N_{f} g^{4} \sigma^{2}}{8 \pi^{2}}\left[1+4 \ln \left(\frac{\sigma}{f_{\pi}}\right)\right] .
\end{gathered}
$$

These equations are then added to the gap equations and change the vacuum parameters $\lambda, m^{2}$, and $H$. Note that $\Lambda$ cancels in the determination of the vacuum parameters (case $Q_{\text {th+vac }}$ in Table II), and hence, the grand canonical potential is also independent of the choice of $\Lambda$. This is also the case for an $\mathrm{SU}(3)$ approach $[23,36,49]$.

\section{THE 2PI FORMALISM}

At finite temperature, perturbative expansion in powers of the coupling constant breaks down due to infrared divergencies, and an approach for the mesonic fields via the path integral formalism leads to difficulties, because at low momentum, spontaneous symmetry breaking, for instance, leads to quasiparticle exitations with imaginary energies [26,27,29].

These difficulties can be circumvented utilizing the Cornwall-Jackiw-Toumboulis (CJT) [40] or, more commonly, the 2PI formalism, which is understood as a relativistic generalization of the Luttinger Ward formalism [50,51]. The 2PI formalism can be viewed as a prescription for computing the effective action of a theory, where the stationary conditions are the Greens functions and the effective action corresponds to the effective potential [40]. However, the in-medium masses of the $\sigma$ - and the $\pi$-meson can then be solved self-consistently $[26,27]$. The starting point is the Lagrangian from the $\mathrm{O}(\mathrm{N})$ model

$$
\begin{gathered}
\mathcal{L}_{O(N)}=\frac{1}{2}\left(\partial_{\mu} \sigma \partial^{\mu} \sigma+\partial_{\mu} \vec{\pi} \partial^{\mu} \vec{\pi}\right) \\
+\frac{\lambda}{N}\left(\left(\sigma^{2}+\vec{\pi}^{2}\right)-v^{2}\right)^{2}-H \sigma
\end{gathered}
$$

where $N=4$ in our calculations to remain in contact with QCD. For $H=0$ and $m^{2} \geq 0, \mathcal{L}_{O(N)}$ is invariant under $\mathrm{O}(\mathrm{N})$ rotations of the fields, whereas for $H=0$ and $m^{2} \leq 0$ this symmetry is spontaneously broken down to $\mathrm{O}(\mathrm{N}-1)$, with N-1 Goldstone bosons, which are the pions. Pions become massive for the explicit symmetry breaking term $H \geq 0$. Spontaneous breaking of a symmetry leads to a nonvanishing vacuum expectation value for the $\sigma$ field, which is the pion decay constant $f_{\pi}=92.4 \mathrm{MeV}$.

The grand canonical potential can be derived via the generating functional for the respective Greens functions [40], which, in the presence of the two sources $J$ and $K$, is given as 


$$
Z[J, K]=e^{\mathcal{W}[J, K]}=\int \mathcal{D} \phi e^{\left(\phi J+\frac{1}{2} \phi K \phi+I[\phi]\right)},
$$

with $\mathcal{W}[J, K]$ as the generating functional for the connected Greens functions. $I[\phi]=\int_{x} \mathcal{L}$ is the classical action with $\mathcal{L}=\mathcal{L}_{m}+U(\sigma, \vec{\pi})$ from Eq. (7). Throughout this article we stick to the shorthand notation

$$
\int_{x} F(x)=\int_{0}^{\beta} d \tau \int d^{3} \vec{k} F(\tau, \vec{k})
$$

for the corresponding integrals, where $\mathcal{F}$ is the appropriate distribution function.

The expectation values for the one- and two-point functions according to $[26,27]$ are

$$
\begin{gathered}
\frac{\delta \mathcal{W}[J, K]}{\delta J(x)} \equiv \bar{\phi}(x) \\
\frac{\delta \mathcal{W}[J, K]}{\delta K(x, y)} \equiv \frac{1}{2}(G(x, y)+\bar{\phi}(x) \bar{\phi}(y)) .
\end{gathered}
$$

To obtain the effective action, a double Legendre transformation needs to be performed, leading to

$$
\Gamma[\bar{\phi}, G]=\mathcal{W}[J, K]-\bar{\phi} J-\frac{1}{2} \bar{\phi} K \bar{\phi}-\frac{1}{2} G K,
$$

where $G K \equiv \int_{x, y} G(x, y) K(y, x)$. Thus,

$$
\begin{gathered}
\frac{\delta \Gamma[\bar{\phi}, G]}{\delta \bar{\phi}}=J(x)-\int_{y} K(x, y) \phi(y), \\
\frac{\delta \Gamma[\bar{\phi}, G]}{\delta G(x, y)}=-\frac{1}{2} K(x, y) .
\end{gathered}
$$

For vanishing sources $J(x)=K(x, y)=0$, the stationary conditions then read

$$
\begin{aligned}
& \left.\frac{\delta \Gamma[\bar{\phi}, G]}{\delta \bar{\phi}}\right|_{\bar{\phi}=\varphi, G=\mathcal{G}}=0, \\
& \left.\frac{\delta \Gamma[\bar{\phi}, G]}{\delta G(x, y)}\right|_{\bar{\phi}=\varphi, G=\mathcal{G}}=0,
\end{aligned}
$$

where Eq. (37) corresponds to a Schwinger-Dyson equation for the dressed propagator. The effective action according to [40] is

$$
\begin{aligned}
\Gamma[\bar{\phi}, G]= & I[\bar{\phi}]-\frac{1}{2} \operatorname{Tr}\left(D^{-1} G-1\right) \\
& -\frac{1}{2} \operatorname{Tr}\left(\ln G^{-1}\right)+\Gamma_{2}[\bar{\phi}, G]
\end{aligned}
$$

with $D^{-1}$ as the inverse tree-level propagator and $G$ as the full propagator. $\Gamma_{2}[\bar{\phi}, G]$ represents the sum of all two particle irreducible diagrams; see Fig. 2, where all lines represent full propagators $G$. In momentum space

$$
D^{-1}(k, \bar{\phi})=-k^{2}+U^{\prime \prime}(\bar{\phi}),
$$

and the full propagator is

$$
G_{\sigma, \pi}(k)=\frac{1}{-k^{2}+\bar{m}_{\sigma, \pi}^{2}} .
$$

The self-energy is assumed to be momentum independent; however, there is a two-loop diagram with a quark propagator and a meson propagator, which would give a momentum dependent self-energy contribution. For simplicity, we ignore this contribution and leave it for future work because the numerical effort is beyond the scope of the present investigation.

For constant fields $\bar{\phi}(x)=\bar{\phi}$ and homogenous systems, the effective potential is $[26,27,40,41]$

$$
\begin{aligned}
\Omega[\bar{\phi}, G]= & U(\bar{\phi})+\frac{1}{2} \int_{k} \ln G^{-1}(k) \\
& +\frac{1}{2} \int_{k}\left[D^{-1}(k, \bar{\phi}) G(k)-1\right]+\Omega_{2} .
\end{aligned}
$$

Here, $\Omega_{2} \equiv-T \cdot \Gamma_{2}[\bar{\phi}, G] / V, V$ being the 3 -volume of the system. The 2 PI potential reads

$$
\begin{aligned}
\Omega_{2 P I}\left(\phi, G_{\sigma, \pi}\right)= & \frac{1}{2} m^{2} \phi^{2}+\frac{1}{4} \lambda \phi^{4}-H \phi \\
& +\frac{1}{2} \int_{k}\left[\ln G_{\sigma}^{-1}(k)+D_{\sigma}^{-1}(k, \phi) G_{\sigma}(k)-1\right] \\
& +\frac{3}{2} \int_{k}\left[\ln G_{\pi}^{-1}(k)+D_{\pi}^{-1}(k, \phi) G_{\pi}(k)-1\right] \\
& +\Omega_{2},
\end{aligned}
$$

with the two-loop contribution to the potential

$$
\begin{aligned}
\Omega_{2}= & \frac{3 \lambda}{4}\left[\int_{k} G_{\sigma}(k)\right]^{2}+\frac{15 \lambda}{4}\left[\int_{k} G_{\pi}(k)\right]^{2} \\
& +\frac{3 \lambda}{2}\left[\int_{k} G_{\sigma}(k)\right]\left[\int_{k} G_{\pi}(k)\right] .
\end{aligned}
$$

The respective diagrammatic expressions for the potential from Eq. (43) are shown in Figs. 1 and 2.

The gap equations obtained via Eqs. (14) for the meson fields read

$$
\begin{array}{r}
H=\phi\left[m^{2}+\lambda\left(\phi^{2}+3 F\left(\bar{m}_{\sigma}, T\right)+3 F\left(\bar{m}_{\pi}, T\right)\right)\right], \\
\bar{m}_{\sigma}=m^{2}+\lambda\left[3 \phi^{2}+3 F\left(\bar{m}_{\sigma}, T\right)+3 F\left(\bar{m}_{\pi}, T\right)\right], \\
\bar{m}_{\pi}=m^{2}+\lambda\left[\phi^{2}+F\left(\bar{m}_{\sigma}, T\right)+5 F\left(\bar{m}_{\pi}, T\right)\right] .
\end{array}
$$




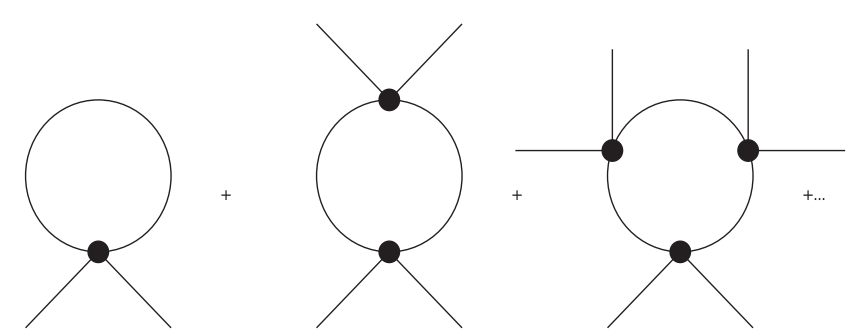

FIG. 1. 1-PI loops contributing to the effective potential in Eq. (42), i.e. Eq. (43) without $\Omega_{2}$ from Eq. (44). (a)

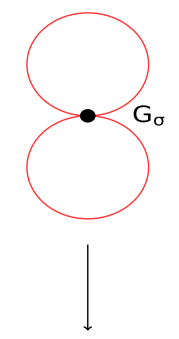

(b)

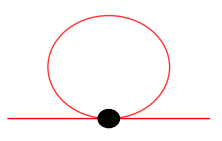

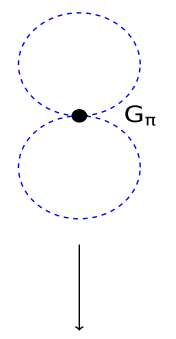

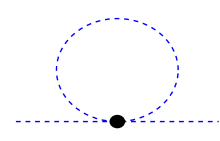

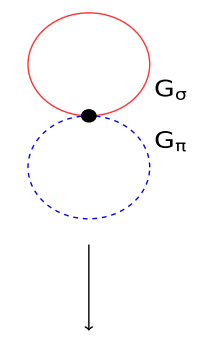

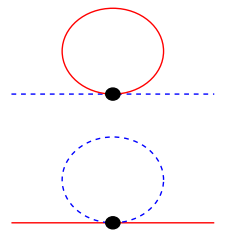

FIG. 2. (a) Two-loop Hartree contributions, Eq. (44), to the CJT effective potential $\left(\Omega_{2}\right)$. The solid red line corresponds to $G_{\sigma}$, whereas the dashed blue line corresponds to $G_{\pi}$. The right-most diagram stands for the last term in Eq. (43). (b) Tadpole contribution to the self-energy, obtained by cutting a line.

Herein the function

$$
\begin{aligned}
F\left(\bar{m}_{\sigma, \pi}, T\right) & =F_{T}\left(\bar{m}_{\sigma, \pi}, T\right)+F_{\mathrm{vac}}\left(\bar{m}_{\sigma, \pi}, T\right) \\
& =\int \frac{d^{3} \vec{k}}{(2 \pi)^{3}} \frac{1}{\sqrt{\vec{k}^{2}+\bar{m}_{\sigma, \pi}^{2}}} \cdot\left[\frac{1}{e^{\beta \sqrt{\bar{k}^{2}+\bar{m}_{\sigma, \pi}^{2}}}-1}+\frac{1}{2}\right]
\end{aligned}
$$

displays the temperature dependence including the vacuum contribution [26]. For more details on the calculation, see $[26,27,40,41]$. The vacuum parameters are listed in Table II. Note that for $J(x)=K(x, y)=0$ and for $\bar{\phi}(x)=\bar{\phi}$, it is justified to combine the potentials from the quarks, i.e. Eq. (9), with the potential from the mesons, i.e. Eq. (43), to evaluate the potentials independently.

Note that on the two-loop level there exist two more diagrams which were also not taken into account and which are connected to the three particle vertices $\lambda \sigma^{3}$ and $\lambda \pi^{2} \sigma$. These depend explicitly on the boson fields and introduce an additional momentum dependence in the SchwingerDyson equations Eqs. (2), (3), and (4), which makes their solution far more complicated and which would violate the ansatz Eq. (41). Our Schwinger-Dyson equations for the full propagators contain no momentum dependence. Thus, our equations are simple gap equations [Eqs. (45)-(47)] for the masses of the $\sigma$ and the $\vec{\pi}$ meson and imply that twoloop diagrams containing quarks and mesons are forbidden. This is in accordance with the general considerations briefly discussed in Sec. II.

\section{A. Regularization for the meson fields}

We use the dimensional regularization procedure for meson fields [52]. Whereas for the quark fields we added a counter term to the Lagrangian, for the meson fields it is sufficient to just add a correction to the mass term, $\delta m$, since no higher order diagrams are considered. The correction to the naked mass is calculated to be $[26,28]$

$$
\delta m^{2}=-\frac{\lambda m^{2}}{16 \pi^{2} \epsilon}-\frac{\lambda m^{2}}{32 \pi^{2}} \ln \left(\frac{4 \pi \mu^{2} e}{m^{2} e^{\gamma}}\right)+\mathcal{O}\left(\epsilon^{2}\right) .
$$

Here, $\mu$ plays the role of $\Lambda$ from the quark fields, i.e. an arbitrary renormalization scale parameter.

The procedure is equivalent to the one for the quark fields [41], utilizing the $\overline{\mathrm{MS}}$ scheme. The renormalized vacuum contribution from Eq. (48) finally reads

$$
\begin{aligned}
F_{\mathrm{vac}}\left(\bar{m}_{\sigma, \pi}\right) & =\int \frac{d^{3} \vec{k}}{(2 \pi)^{3}} \frac{1}{2 \sqrt{\vec{k}^{2}+\bar{m}_{\sigma, \pi}^{2}}} \\
& =-\frac{\bar{m}_{\sigma, \pi}}{16 \pi^{2}}\left[1+\ln \left(\frac{\mu^{2}}{\bar{m}_{\sigma, \pi}}\right)\right] \equiv F_{d r}\left(\bar{m}_{\sigma, \pi}\right) .
\end{aligned}
$$

Again, the vacuum parameters are given in Table II.

\section{COMBINING INTERACTIONS BETWEEN QUARKS AND MESONS}

Since the grand canonical potential is an intensive quantity, it is additive and so are the respective gap equations of the corresponding sectors, obtained in each case with Eq. (14). This section now combines both approaches to a unified set of equations. First, we will treat the thermal contributions only, whereas in the following we include the vacuum fluctuations from the quark fields. The potential is a sum of the independent potentials

$$
\Omega_{\mathrm{QAM}}^{\mathrm{th}}=\Omega_{\bar{q} q}^{\mathrm{th}}+\Omega_{2 P I}\left(\phi, G_{\sigma, \pi}\right) .
$$

Here, $\Omega_{\mathrm{QAM}}^{\text {th }}$ is the thermal part of the combined grand canonical potential of quarks and mesons (QAM).

\section{A. Regularization for the combined approach}

As mentioned above, all relevant quantities are additive and so are the vacuum contributions. Hence, there is no need to regularize and renormalize anew. Both equations for the divergent vacuum contributions, Eqs. (24) and (50), 
can be merged into a single set of gap equations. The potential is the sum of the independent potentials, i.e. Eqs. (9) and (43). The tree-level potential, Eq. (8), appears only once,

$$
\Omega_{\mathrm{QAM}}=\Omega_{\bar{q} q}^{\mathrm{th}}+\Omega_{\bar{q} q}^{d r}+\Omega_{2 P I}\left(\phi, G_{\sigma, \pi}\right) .
$$

The vacuum parameters $\lambda, m^{2}$, and $H$, obtained by solving Eq. (14) are determined to be

$$
\begin{gathered}
\lambda=\frac{m_{\sigma}^{2}+m_{\pi}^{2}+\frac{N_{c} N_{f}}{8 \pi^{2}} g^{4} \sigma^{2}\left[6+8 \ln \left(\frac{g \sigma}{\Lambda}\right)\right]}{2\left(F_{d r}\left(\bar{m}_{\sigma}\right)-F_{d r}\left(\bar{m}_{\pi}\right)+\sigma^{2}\right)}, \\
m^{2}=\frac{N_{c} N_{f}}{8 \pi^{2}} g^{4} \sigma^{2}\left[7+12 \ln \left(\frac{g \sigma}{\Lambda}\right)\right] \\
-3 \lambda\left(F_{d r}\left(\bar{m}_{\sigma}\right)+F_{d r}\left(\bar{m}_{\pi}\right)\right)+m_{\sigma}^{2}-3 \lambda \sigma^{2}, \\
H=-\frac{N_{c} N_{f}}{8 \pi^{2}} g^{4} \sigma^{3}\left[1+4 \ln \left(\frac{g \sigma}{\Lambda}\right)\right] \\
+3 \lambda \sigma\left(F_{d r}\left(\bar{m}_{\sigma}\right)+F_{d r}\left(\bar{m}_{\pi}\right)\right)+\sigma\left(m^{2}+\lambda \sigma^{2}\right),
\end{gathered}
$$

and the corresponding gap equations read

$$
\begin{aligned}
\frac{\partial \Omega_{\mathrm{QAM}}}{\partial \sigma}= & -\frac{N_{c} N_{f}}{8 \pi^{2}} g^{4} \sigma^{3}\left[1+4 \ln \left(\frac{g \sigma}{\Lambda}\right)\right] \\
& +3 \lambda \sigma\left(F\left(\bar{m}_{\sigma}\right)+F\left(\bar{m}_{\pi}\right)\right)+m^{2} \sigma+\lambda \sigma^{3}=H,
\end{aligned}
$$

$$
\begin{aligned}
\frac{\partial^{2} \Omega_{\mathrm{QAM}}}{\partial \sigma^{2}}= & -\frac{N_{c} N_{f}}{8 \pi^{2}} g^{4} \sigma^{2}\left[7+12 \ln \left(\frac{g \sigma}{\Lambda}\right)\right] \\
& +3 \lambda\left(F\left(\bar{m}_{\sigma}\right)+F\left(\bar{m}_{\pi}\right)\right)+m^{2}+3 \lambda \sigma^{2}=m_{\sigma}^{2},
\end{aligned}
$$

$$
\begin{aligned}
\frac{\partial^{2} \Omega_{\mathrm{QAM}}}{\partial \pi^{2}}= & -\frac{N_{c} N_{f}}{8 \pi^{2}} g^{4} \sigma^{2}\left[1+4 \ln \left(\frac{g \sigma}{\Lambda}\right)\right] \\
& +\lambda\left(F\left(\bar{m}_{\sigma}\right)+F\left(\bar{m}_{\pi}\right)\right)+m^{2}+\lambda \sigma^{2}=m_{\pi}^{2} .
\end{aligned}
$$

Unfortunately, these equations leave us with the possibility of having two renormalization scales, one from the quarkquark contribution, $\Lambda$, and one hidden in $F\left(\bar{m}_{\sigma \pi}\right)$, namely, $\mu$ [see Eq. (50)]. The vacuum parameters are listed in Table II.

\section{RESULTS FOR THE RENORMALIZED QUARK FIELDS}

The upper panel of Fig. 3 shows the order parameter $\sigma$ as a function of the temperature for three different vacuum sigma meson masses $m_{\sigma}^{\mathrm{vac}}$, neglecting (denoted in the figures as "th.") and including (denoted in the figures as "vac.") the vacuum term of the quarks. This corresponds to the cases $Q_{\mathrm{th}}$ and $Q_{\mathrm{th}+\mathrm{vac}}$ in Table II.
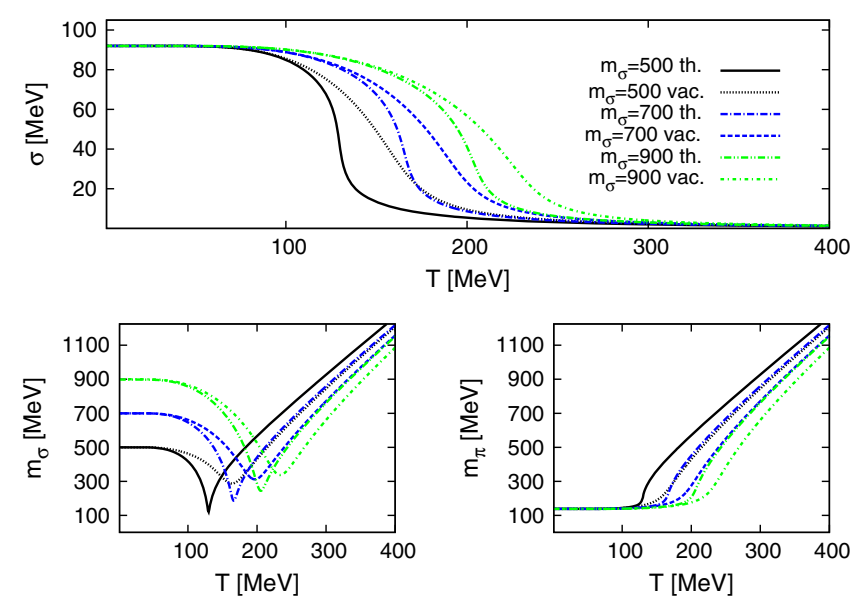

FIG. 3. $\sigma$ condensate as a function of temperature for zero chemical potential without (denoted as "th.") and with vacuum contribution (denoted as "vac.") for three different values of the vacuo sigma meson mass $m_{\sigma}^{\mathrm{vac}}$ shown in the upper panel. The lower panels show the in-medium masses of the $\sigma$ and the $\pi$.

We find that with increasing vacuum sigma meson mass $m_{\sigma}^{\mathrm{vac}}$ the phase transition in the thermal case is shifted to higher temperatures and becomes slightly more crossoverlike, whereas smaller values of $m_{\sigma}^{\mathrm{vac}}$ lead to a behavior close to a first order phase transition, which is not achieved even for our lowest choice of $m_{\sigma}^{\mathrm{vac}}=500 \mathrm{MeV}$. The curves containing the vacuum contribution show the same behavior; only the trends are noticeably more crossoverlike and hence shifted to higher transition temperatures with increasing values of $m_{\sigma}^{\mathrm{vac}}$.

The behavior of the order parameter $\sigma$ can be translated to the behavior of the masses as a function of the temperature; see the lower two panels in Fig. 3. The respective minimum of the sigma mass in the lower left panel in Fig. 3 represents the point of the chiral phase transition. From there on, the mass of the sigma and the pion start to be degenerate.

For $m_{\sigma}^{\mathrm{vac}}=500 \mathrm{MeV}$, when neglecting the vacuum term, the sigma and the pion mass come close to the chiral limit. Here, $T=130 \mathrm{MeV}$ and $m_{\sigma}=120 \mathrm{MeV}$; see also Table I, and the pion mass nearly jumps vertically around this temperature. The inclusion of the vacuum contribution for all values of the initial vacuum mass $m_{\sigma}^{\mathrm{vac}}$ leads to a less distinctive decrease of $m_{\sigma}$ toward the chiral transition, going along with a clearly less pronounced minimum, which is also located at higher temperatures and higher $m_{\sigma}$ compared to the respective thermal value, i.e. when neglecting the vacuum term. From the phase transition point on the mass of the pion, which is seen in the lower right panel of Fig. 3, is degenerate to the mass of the sigma. At $T=400 \mathrm{MeV}$, sigma and pion masses of $\sim 1.2 \mathrm{GeV}$ are achieved. The upper panel in Fig. 4 shows the pressure for the three different vacuum sigma meson masses including and neglecting the vacuum term. All curves rise monotonically. In the temperature region 
TABLE I. The minimal mass for the $\sigma$-meson for all three different approaches, i.e. quarks with and without the vacuum term, case $Q_{\text {th } / \text { vac }}$ (Sec. III), mesons with and without the vacuum term, case $M_{\mathrm{th} / \mathrm{vac}}(\mathrm{Sec} . \mathrm{IV})$, and quarks and mesons combined with and without the vacuum term for the quark fields, $Q_{\text {th } / v a c}+$ $M_{\text {th }}($ Sec. V). All values are given in MeV.

\begin{tabular}{|c|c|c|c|c|c|c|}
\hline \multirow[b]{2}{*}{$m_{\sigma}^{\mathrm{vac}}$} & \multicolumn{2}{|c|}{$Q_{\text {th } / \mathrm{vac}}$} & \multicolumn{2}{|c|}{$M_{\text {th } / \mathrm{vac}}$} & \multicolumn{2}{|c|}{$Q_{\mathrm{th} / \mathrm{vac}}+M_{\mathrm{th}}$} \\
\hline & $T$ & $m_{\sigma}$ & $T$ & $m_{\sigma}$ & $T$ & $m_{\sigma}$ \\
\hline $500_{(\text {th })}$ & 130 & 120 & 230 & 290 & 118 & 150 \\
\hline $500_{(\mathrm{th}+\mathrm{vac})}$ & 163 & 287 & 260 & 320 & 166 & 285 \\
\hline $700_{(\mathrm{th})}$ & 165 & 185 & 238 & 324 & 143 & 214 \\
\hline $700_{(\mathrm{th}+\mathrm{vac})}$ & 198 & 310 & 305 & 414 & 185 & 316 \\
\hline $900_{(\mathrm{th})}$ & 205 & 243 & 245 & 355 & 165 & 267 \\
\hline $900_{(\mathrm{th}+\mathrm{vac})}$ & 233 & 336 & 360 & 510 & 201 & 344 \\
\hline
\end{tabular}

$100 \mathrm{MeV} \leq T \leq 350 \mathrm{MeV}$, the curves separate, and the pressure becomes smaller with increasing value of the vacuum sigma meson mass. The inclusion of vacuum fluctuations intensifies this trend at a given $m_{\sigma}^{\mathrm{vac}}$ so that the pressure within this temperature range is the smallest for high $m_{\sigma}^{\mathrm{vac}}$ and for inclusion of the self-energy. The higher the vacuum mass of the sigma, the less pronounced are the effects from the inclusion of the vacuum fluctuations. For the smallest value of the initial vacuum sigma meson mass $m_{\sigma}^{\mathrm{vac}}=500 \mathrm{MeV}$ and neglecting the vacuum contribution, the quarks reach the Stefan-Boltzmann limit (SB limit in the figures) at the lowest temperature, whereas the inclusion of the vacuum contribution at $m_{\sigma}^{\mathrm{vac}}=$ $500 \mathrm{MeV}$ pushes down the pressure within the temperature region $100 \mathrm{MeV} \leq T \leq 350 \mathrm{MeV}$. This statement is valid for all $m_{\sigma}^{\mathrm{vac}}$ and can be understood as an intrinsic property of the self-energy. The quarks are more massive for high $m_{\sigma}^{\mathrm{vac}}$. This matches the statement concerning the respective

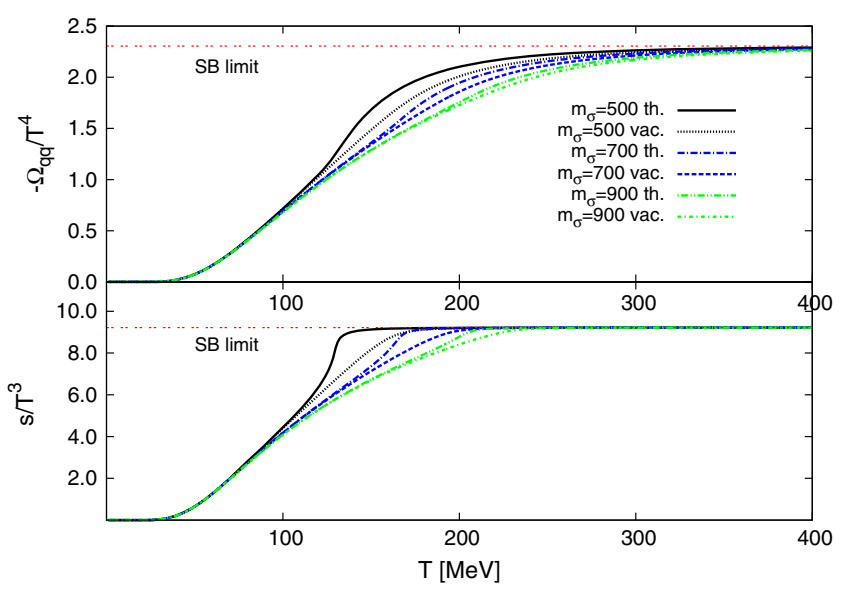

FIG. 4. Pressure, divided by $T^{4}$ as a function of temperature for zero chemical potential without (denoted as "th.") and with vacuum contribution (denoted as "vac.") for three different values of the initial vacuum sigma meson mass $m_{\sigma}^{\mathrm{vac}}$ shown in the upper plot. The lower plot shows the entropy density $s$ divided by $T^{3}$ as a function of the temperature. The SB limit represents the Stefan Boltzmann limit.

mass spectrum of the sigma and the pion at high temperature and can also be observed from the behavior of the order parameter $\sigma$. Recalling that the effective mass of the quarks is generated through the coupling $g$ and the fields [see Eq. (13)], this conclusion is not surprising.

The lower plot in Fig. 4 shows the entropy density divided by $T^{3}$ of the three different initial sigma meson masses $m_{\sigma}^{\text {vac }}$ including and neglecting the vacuum contributions. The entropy density for small $m_{\sigma}^{\mathrm{vac}}$ and without the vacuum term has higher values at a given temperature compared to that in the cases with high initial vacuum mass $m_{\sigma}$ and the inclusion of self-energy. This feature stems from the fact that as the disorder in the system gets larger, the

TABLE II. The parameters $\lambda, m^{2}$, and $H$ for all considered cases. Thermal quarks are labeled $Q_{\text {th }}$, including the vacuum term for the quark fields, which is labeled $Q_{\text {th+vac }}$. Thermal mesons without the vacuum term are labeled $M_{\text {th }}$ and those with the vacuum term $M_{\text {th }+ \text { vac }}$. The approach combining quarks and mesons without the vacuum term is labeled $Q_{\text {th }}+M_{\text {th }}$. For these cases the sigma meson mass is $m_{\sigma}^{\mathrm{vac}}=500 \mathrm{MeV}$. The combination of both approaches with the vacuum term only for the quark fields is labeled $Q_{\mathrm{th}+\mathrm{vac}}+M_{\mathrm{th}}$ and that with the vacuum term in both approaches $Q_{\mathrm{th}+\mathrm{vac}}+M_{\mathrm{th}+\mathrm{vac}}$. Here $m_{\sigma}^{\mathrm{vac}}=550 \mathrm{MeV}$ for the different choices of the renormalization scale, which is given in MeV. $\lambda$ is dimensionless, $m^{2}$ is in $\mathrm{MeV}^{2}$, and $H$ is given in $\mathrm{MeV}^{3}$.

\begin{tabular}{lcccccc}
\hline \hline Case & $m_{\sigma}^{\mathrm{vac}}$ & $\Lambda$ & $\mu$ & $\lambda$ & $m^{2}$ & $H$ \\
\hline$Q_{\text {th }}$ & 500 & $\ldots$ & $\ldots$ & 16.744 & -122683 & $1.75 \times 10^{6}$ \\
$Q_{\text {th }+\mathrm{vac}}$ & 500 & $\ldots$ & $\ldots$ & 42.521 & -268130 & $1.75 \times 10^{6}$ \\
$M_{\text {th }}$ & 500 & $\ldots$ & $\ldots$ & 16.744 & -122683 & $1.75 \times 10^{6}$ \\
$M_{\text {th }+\mathrm{vac}}$ & 500 & $\ldots$ & 333.591 & 16.11 & -90449 & $2.74 \times 10^{6}$ \\
$Q_{\text {th }}+M_{\text {th }}$ & 500 & $\ldots$ & $\ldots$ & 16.744 & -122683 & $1.75 \times 10^{6}$ \\
$Q_{\text {th }+\mathrm{vac}}+M_{\text {th }}$ & 550 & 1033 & $\ldots$ & 0.0268 & -268130 & $1.75 \times 10^{6}$ \\
$Q_{\text {th }+\mathrm{vac}}+M_{\text {th }+\mathrm{vac}}: \Lambda=\mu$ & 550 & 1033 & 1033 & 0.013 & -268148 & $1.77 \times 10^{6}$ \\
$Q_{\text {th }+\mathrm{vac}}+M_{\text {th }+\mathrm{vac}}: \Lambda \neq \mu$ & 550 & 900 & 333.591 & 4.583 & -258959 & $2.03 \times 10^{6}$ \\
$Q_{\text {th }+\mathrm{vac}}+M_{\text {th }+\mathrm{vac}}: \Lambda \neq \mu$ & 550 & 1000 & 333.591 & 1.099 & -265930 & $1.82 \times 10^{6}$ \\
$Q_{\text {th }+\mathrm{vac}}+M_{\text {th }+\mathrm{vac}}: \Lambda \neq \mu$ & 550 & 1100 & 333.591 & -2.052 & -272236 & $1.62 \times 10^{6}$ \\
\hline \hline
\end{tabular}


more free are the quarks. Remember, that the higher vacuum value $m_{\sigma}^{\mathrm{vac}}$, the higher is the temperature, where quarks reach the chiral limit, leading to heavier quarks at intermediate temperatures. The inclusion of the vacuum energy term amplifies this effect, for low $m_{\sigma}^{\mathrm{vac}}$ more significantly than for large $m_{\sigma}^{\mathrm{vac}}$.

\section{RESULTS FOR THE COMBINED APPROACH}

At first, we neglect the vacuum contribution from the quark and meson fields, which is denoted as (usual) "th." $\left(Q_{\text {th }}+M_{\text {th }}\right.$ in Table II) by setting $F_{d r}\left(\bar{m}_{\sigma, \vec{\pi}}\right)=0$. Even when excluding the mesonic vacuum contribution, the dependence on the quark renormalization scale $\Lambda$ does not vanish contrary to the case for the quark fields only; see Sec. III A. This is due to the contribution from $\Omega_{2 P I}$ and corresponds to the case $Q_{\mathrm{th}+\mathrm{vac}}+M_{\text {th }}$ in Table II. We choose a value of $\Lambda=1033 \mathrm{MeV}$ due to reasons which will become clear in Sec. VII B, where we discuss the dependence on both renormalization scales $\left(Q_{\mathrm{th}+\mathrm{vac}}+M_{\mathrm{th}+\mathrm{vac}}\right.$ in Table II).

\section{A. Results for the combined approach 1: Quark vacuum energy}

The upper panel in Fig. 5 shows the order parameter $\sigma$ as a function of the temperature within the combined approach for the choice of the renormalization scale $\Lambda=1033 \mathrm{MeV}$. As expected, the larger the value of the initial vacuum sigma meson mass $m_{\sigma}^{\mathrm{vac}}$, the further is the curve shifted to higher temperatures. The vacuum contribution leads to the same trend as when raising the initial value of $m_{\sigma}^{\mathrm{vac}}$ so that a
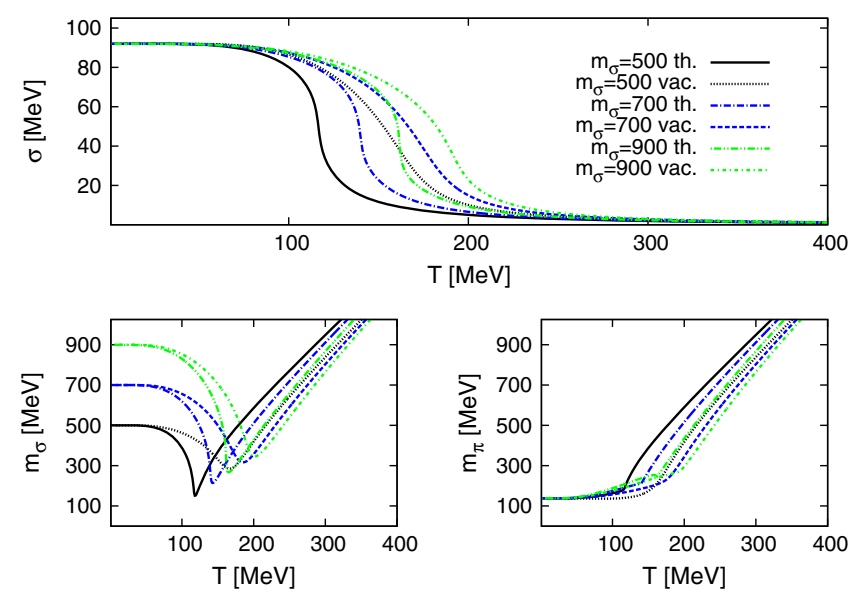

FIG. 5. $\sigma$ condensate in the combined approach as a function of temperature for zero chemical potential without (denoted as "th.") and with quark vacuum contribution (denoted as "vac.") for three different values of the initial vacuo sigma meson mass $m_{\sigma}^{\text {vac }}$ shown in the upper panel. The lower panels show the masses of the sigma and the pion as a function of temperature. The value of the quark renormalization scale has been chosen to be $\Lambda=1033 \mathrm{MeV}$. high vacuum mass $m_{\sigma}^{\mathrm{vac}}$ accompanied with the inclusion of the vacuum energy leads to the highest phase transition temperature. The sigma meson mass as a function of the temperature is shown in the lower panel of Fig. 5. The minima of the sigma meson mass curve, indicating the critical phase transition temperature $T_{c}$, are closer to the values from the case $Q_{\text {th }}$ than from the case $M_{\text {th }}$; see Table I. This statement is valid in the thermal cases as well when including the fermion vacuum term $Q_{\text {vac }}$. For low $m_{\sigma}^{\mathrm{vac}}$, the minima values are relatively close to the ones from the case $Q_{\mathrm{th}}$. Increasing $m_{\sigma}^{\mathrm{vac}}$ shifts the minima, indicating that the meson contribution gains influence.

The behavior of the pion mass can be seen in the lower right panel in Fig. 5. The curves seem to be a combination of the pion mass spectrum from the case $Q_{\text {th }}$ and the one from the case $M_{\text {th }}$, where also the quark contribution dominates. For larger values of $m_{\sigma}^{\mathrm{vac}}$, the pion mass starts to increase at lower temperatures, which is a feature seen for the case $M_{\mathrm{th}}$. This again emphasizes the statement that for larger sigma meson mass the meson contributions gain influence within the combined approach. In conclusion, the quarks are dominant in the combined approach. The influence of the meson fields leads to a slightly steeper decrease of the order parameter $\sigma$ indicating a trend toward a first order phase transition, which is not achieved. Both mass spectra in Fig. 5 reach $\sim 1.2 \mathrm{GeV}$ at $T=$ $400 \mathrm{MeV}$ as is the case for the cases $Q_{\text {th }}$ and $Q_{\text {vac }}$ exclusively. In comparison, the mass spectra in the cases $M_{\text {th }}$ and $M_{\text {vac }}$ reach $500 \leq m_{\sigma, \pi} \leq 700 \mathrm{MeV}$, depending on the initial value of $m_{\sigma}^{\mathrm{vac}}$. The vacuum parameters $\lambda, m^{2}$, and $H$ [Eqs. (53)-(55)] for the case $Q_{\mathrm{th}+\mathrm{vac}}+M_{\mathrm{th}}$ are listed in Table II.

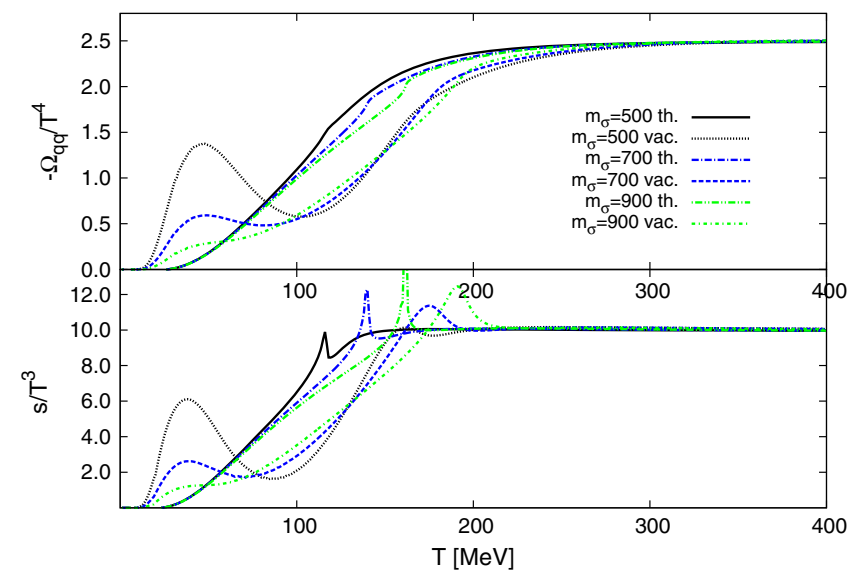

FIG. 6. Negative of the potential, i.e. the pressure, divided by $T^{4}$ as a function of temperature without (denoted as "th.") and with vacuum contribution (denoted as "vac.") for three different values of the initial vacuo sigma meson mass $m_{\sigma}^{\text {vac }}$ shown in the upper panel. The lower panel depicts the entropy density $s$ as a function of temperature. Some curves clearly show maxima and minima. 
The pressure of the combined system divided by $T^{4}$ provided by the SU(2) quark-meson model and the CJT formalism is shown in the upper panel in Fig. 6.

All curves for the case without the vacuum term start to rise significantly at $T \simeq 30 \mathrm{MeV}$, whereas the inclusion of the vacuum term causes the pressure to rise at $T \simeq 20 \mathrm{MeV}$. This behavior results from the mesonic contributions. The curves show distinct extrema, less pronounced with larger $m_{\sigma}^{\mathrm{vac}}$, located around $T \simeq 45 \mathrm{MeV}$. This clearly is correlated to the influence of the vacuum term leading to a higher pressure at a given temperature compared to the case without the vacuum term. In the combined approach, this leads to distinct extrema, indicating the dominance of the meson contribution at low temperature. It is important to note that these extrema are not instabilities since the pressure itself is a monotonically rising function, and so is the entropy density, which is seen in the lower panel in Fig. 6. Neglecting the vacuum contribution, the curves also exhibit a nontrivial behavior within the temperature range $100 \leq T \leq 180 \mathrm{MeV}$, again leading to very distinctive maxima in the entropy density. The entropy density curves without the vacuum term rise approximately linear at low temperature. For $m_{\sigma}^{\mathrm{vac}}=500 \mathrm{MeV}$, a maximum at $T=116 \mathrm{MeV}$ and $s / T^{3}=9.85$ can be observed, which can be traced back to the hardly visible change of slope in the pressure in the upper panel. The higher the vacuum sigma meson mass, the more pronounced are the maxima in $s / T^{3}$. This occurs in all cases considered at the phase transition. These peaks arise from the fact that the pressure has a considerable change of slope at the chiral phase transition temperature. A possible explanation of having two maxima might be that the change of the relativistic degrees of freedom (d.o.f.) occurs in two different temperature regions. One can interpret these pronounced peaks as an intermediate sudden increase in relativistic d.o.f. or as an field energy contribution. Note also, that an entropy jump as in a first-order phase transition is not observed.

Table I shows the minimal value of the sigma meson mass in the medium for the cases $Q_{\mathrm{th}}, Q_{\mathrm{vac}}, M_{\mathrm{th}}, M_{\mathrm{vac}}$, and for $Q_{\mathrm{th} / \mathrm{vac}}+M_{\mathrm{th}}$. With or without the vacuum term, the minima of the combined approach are closer to the values of the thermal quarks than to the values for thermal mesons. The impact of the thermal mesons shifts the minima of the combined approach to lower temperatures.

\section{B. Results for the combined approach 2: Dependence on the renormalization scale}

In this section, we explore the impact of having two renormalization scales, one from the quark fields $\Lambda$ and one from the mesonic fields $\mu$. This corresponds to the case $Q_{\mathrm{th}+\mathrm{vac}}+M_{\mathrm{th}+\mathrm{vac}}$ in Table II. In the last subsection, we set $F_{d r}\left(\bar{m}_{\sigma, \vec{\pi}}\right)=0$, omitting the self-energy resulting from the 2PI formalism from the mesonic fields. In this section, we
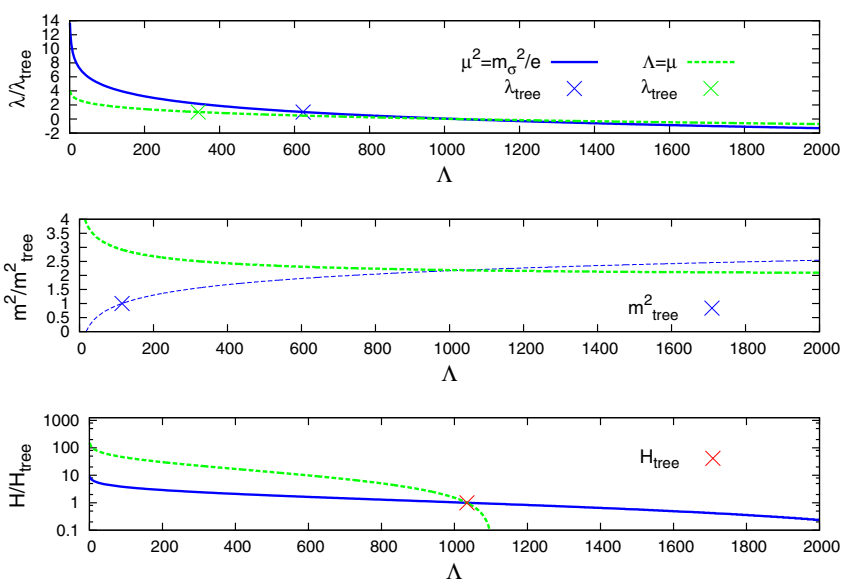

FIG. 7. Vacuum parameters $\lambda, m^{2}$, and $H$, normalized to their respective tree-level value $\left(\lambda \simeq 16.64, m^{2}=-122683 \mathrm{MeV}^{2}\right.$ and $\left.H=1.75 \times 10^{6} \mathrm{MeV}^{3}\right)$, as a function of the quark renormalization scale $\Lambda$. The cross marks the tree-level values.

show that this contribution is negligible for the fields and the mass spectra but not for the thermodynamics, i.e. the respective relativistic d.o.f. First we run the code with one value for the renormalization scale, i.e. setting $\Lambda=\mu$, and in a second approach, we keep $\mu$ fixed at the value used in [28], i.e. $\mu=m_{\sigma} / \sqrt{e}$. We first study the three vacuum parameters $\lambda$ [Eq. (53)], $m^{2}$ [Eq. (54)], and $H$ [Eq. (55)] as a function of the renormalization scale for $\Lambda=\mu$ and for the choice $\mu=m_{\sigma} / \sqrt{e}$, such as to locate the most reasonable renormalization scale value, which turns out to be the one used in the previous section, $\Lambda=1033 \mathrm{MeV}$. The value of the sigma meson mass has been chosen to be at a value of $m_{\sigma}=550 \mathrm{MeV}$. The renormalization scale parameter is naturally placed at the chiral scale $[2,26,27]$, i.e. of the order $1 \mathrm{GeV}$. Setting $\Lambda=\mu$ or even $\mu=m_{\sigma} / \sqrt{e}$, we find reasonable solutions only within the range $850 \leq \Lambda \leq 1150 \mathrm{MeV}$, which we investigate in this section.

Figure 7 shows the coupling $\lambda$, the mass term $\mathrm{m}^{2}$, and the explicit symmetry breaking term $H$ normalized to their respective tree-level values as a function of the renormalization scale with $\Lambda=\mu$ (dotted curve) and with $\mu=$ $m_{\sigma} / \sqrt{e}=333.591 \mathrm{MeV}$ held fixed (solid curve). The respective values are also given in Table II.

The tree-level value for $\lambda$ for the choice $\Lambda=\mu$ is found to be located at $\Lambda=343 \mathrm{MeV}$, which is surprisingly close to $\mu=m_{\sigma} / \sqrt{e} \mathrm{MeV}$. However, for the choice $\mu=m_{\sigma} / \sqrt{e} \mathrm{MeV}$ the tree-level value is located at $\Lambda=623 \mathrm{MeV}$. Note that the two curves in the upper panel intersect at $\Lambda=1033 \mathrm{MeV}$.

The tree-level value of $m^{2}$ for $\Lambda=\mu$ is never reached (middle figure), and when setting $\mu=m_{\sigma} / \sqrt{e} \mathrm{MeV}$, the curve surprisingly increases with $\Lambda$, and the tree-level value is located at $\Lambda=115 \mathrm{MeV}$. These two curves also intersect at $\Lambda=1033 \mathrm{MeV}$. 


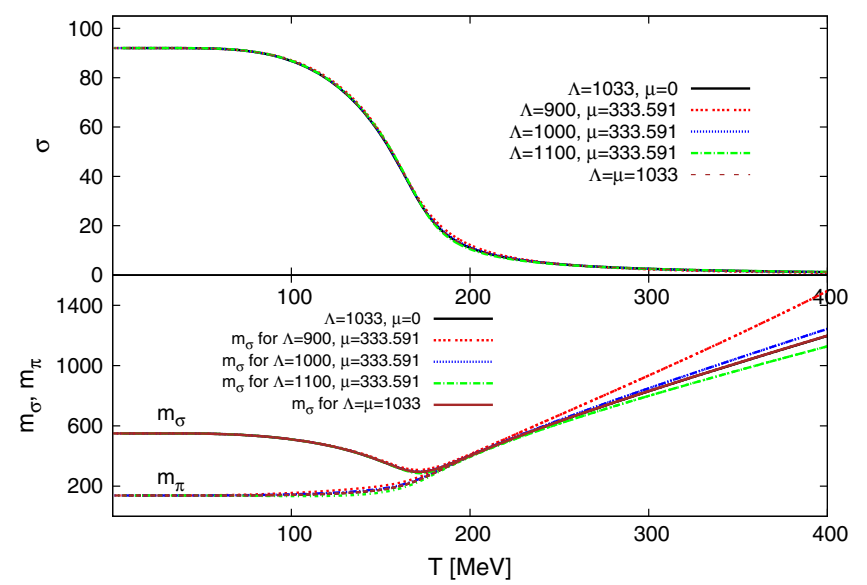

FIG. 8. $\sigma$ condensate as a function of temperature for the renormalization scales $\Lambda=1033 \mathrm{MeV}$ and $\mu=0$ (Sec. VII A), $\Lambda=900,1000,1100 \mathrm{MeV}$ at $\mu=m_{\sigma} / \sqrt{e}$ held fixed, and for $\Lambda=\mu=1033 \mathrm{MeV}$ at $m_{\sigma}=550 \mathrm{MeV}$ is shown in the upper panel. The lower panel shows the sigma and the pion mass spectrum.

The explicit symmetry breaking term $H$, which is responsible for the mass of the pion, is shown normalized to its tree-level value in the lowest panel in Fig. 7. The treelevel value is for both choices $\left(\Lambda=\mu\right.$ and for $\left.\mu=m_{\sigma} / \sqrt{e}\right)$ located at $\Lambda=1033 \mathrm{MeV}$, where these two curves also intersect (which motivates our choice for $\Lambda=\mu=$ $1033 \mathrm{MeV}$ in the previous subsection).

The order parameter $\sigma$ for different renormalization scales is shown in the upper panel in Fig. 8, whereas the lower part shows the mass spectrum of the sigma and the pion.

Figure 8 contains the calculation for only one renormalization scale with $\Lambda=1033 \mathrm{MeV}$ and $\mu=0$ for $m_{\sigma}^{\mathrm{vac}}=$ $550 \mathrm{MeV}$ from Sec. VII A for comparison. For the choice for $\mu$ according to [28], we choose three values of $\Lambda$, and finally we set $\Lambda=\mu=1033 \mathrm{MeV}$. All cases show a crossover phase transition at $T \simeq 165 \mathrm{MeV}$, and there is no notable difference in the order parameter. The different cases for the mass spectrum do not show significant differences up to $T \simeq 250 \mathrm{MeV}$, where the degenerate masses of the sigma and the pion start to have different slopes. It is worth mentioning that the curves are very similar to the curves from the case $Q_{\mathrm{th}}$ or $Q_{\mathrm{vac}}$ and result in similar masses at large temperatures, demonstrating again the dominance of the quark contribution.

The pressure divided by $T^{4}$ as a function of temperature for the renormalization scale choices $\Lambda=$ $1033 \mathrm{MeV}$ and $\mu=0$ (Sec. VII A), $\Lambda=900,1000$, $1100 \mathrm{MeV}$ at $\mu=m_{\sigma} / \sqrt{e}$ held fixed, and for $\Lambda=\mu=$ $1033 \mathrm{MeV}$ at $m_{\sigma}=550 \mathrm{MeV}$ are represented in the upper panel in Fig. 9. All of the curves show two maxima, one at $T \simeq 50 \mathrm{MeV}$ and a smaller one around the phase transition at $T \simeq 165 \mathrm{MeV}$. For $\Lambda=1033 \mathrm{MeV}$ and $\mu=0$, the maximum is located within the same region as that for

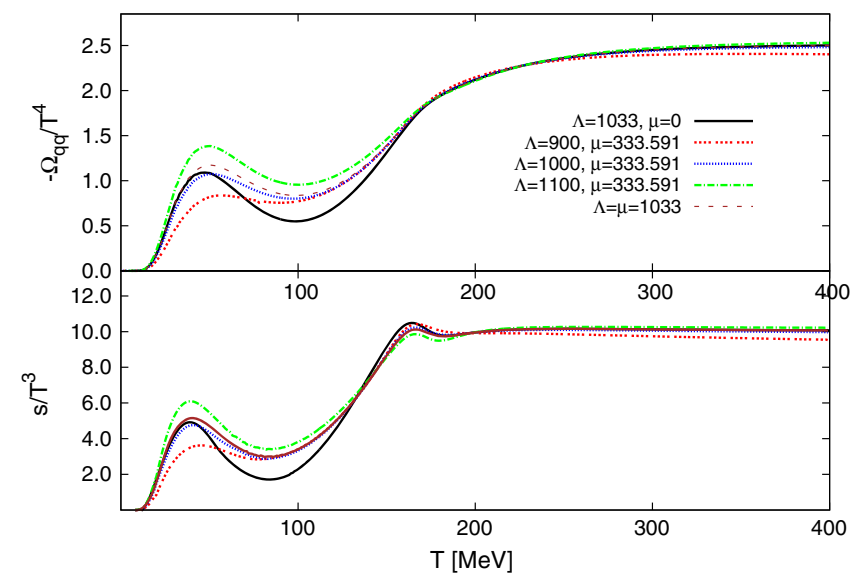

FIG. 9. Pressure, divided by $T^{4}$ as a function of temperature for the renormalization scale choices $\Lambda=1033 \mathrm{MeV}$ and $\mu=0$ (Sec. VII A), $\Lambda=900,1000,1100 \mathrm{MeV}$ at $\mu=m_{\sigma} / \sqrt{e}$ held fixed, and for $\Lambda=\mu=1033 \mathrm{MeV}$ at $m_{\sigma}=550 \mathrm{MeV}$, is shown in the upper panel. The lower panel shows the entropy density $s$ divided by $T^{3}$ as a function of temperature.

two renormalization scales, whereas the minimum is shifted to a considerably lower value of $p / T^{4}$. The second extrema are a result of the contribution from the mesonic fields and change slightly with the choice of the renormalization scale.

\section{CONCLUSIONS}

In this article, we have studied quarks, with the common path integral formalism, and mesons, utilizing the 2PI formalism, within the $\mathrm{SU}(2)$ quark-meson model at zero chemical potential in a combined set of equations.

We investigated the influence of the vacuum fluctuations for different values of the sigma meson mass and for different choices of the renormalization scale parameters on the order parameter, the mass spectra of the sigma and the pion, and for thermodynamical quantities.

The inclusion of the vacuum fluctuations for the quark fields is independent of the renormalization scale $[23,36]$, whereas for the meson fields the dependence on the renormalization scale does not cancel. Inclusion of the vacuum term for the quark fields leads to a distinct shift of the chiral phase transition to higher temperatures. The inclusion of the vacuum contribution turned out to be in both cases not negligible. Within the combined case, we were hence left with the option of having two renormalization scales or one for quarks and mesons.

We investigated separately the vacuum parameters $\lambda, m^{2}$, and $H$ as a function of the quark renormalization scale $\Lambda$ and conclude that the main impact comes from the quark fields. There is a tiny window around $\Lambda \sim 1 \mathrm{GeV}$, where the results are physically reasonable, i.e. close to tree-level values. The fields and the mass spectra showed hardly any difference when varying the renormalization 
scale. It seems that the thermal contributions of the mesons have an influence within the temperature region $50 \leq T \leq 180 \mathrm{MeV}$ for the pressure, which gives rise to peaks within the entropy to temperature ratio. According to lattice QCD calculations, this behavior is clearly unphysical [53] so that only the results for $Q_{\mathrm{th}+\mathrm{vac}}$ and $Q_{\mathrm{th}}+M_{\mathrm{th}}$ are employable. We find that in all cases considered a chiral first order phase transition is not present.

Reference [46] compares the renormalized linear sigma model with the NJL model. Like in our case, a crossover transition has been found for zero chemical potential, and the authors stress the importance of the vacuum field fluctuations to the thermodynamic properties. In Ref. [2], the linear sigma model including the vacuum field fluctuations, containing quark and mesonic d.o.f., has been studied. The quark d.o.f. have been integrated out, and the resulting effective action was linearized around the ground state. Sigma mesons and pions were described as quasiparticles, and their properties were taken into account within the thermodynamic potential. Their parameter choice is similar to ours, and they find a gradual decrease of the chiral condensate, which results in a crossover type transition at temperatures $150 \leq T_{c} \leq 200 \mathrm{MeV}$. Also, the results for the masses are very similar to our results. Their thermodynamical quantities do not show such an influence from the meson fields in the low temperature region. We argue that this feature comes from the 2PI formalism used in our work.

\section{OUTLOOK}

As is the case for the neglected bosonic two-loop diagrams $\lambda(\sigma, \vec{\pi})$, recall Sec. IV, the neglected meson loop with a quark propagator would also be momentum dependent and hence violate our ansatz [Eq. (41)]. It might be interesting to explore the implications of these momentum dependent diagrams in future work, but for simplicity, we ignore this contribution because the numerical effort is beyond the scope of the present investigation. It is, however, not suppressed because it is of the order $\sim N$, but its implementation would make our calculation intractable and the effort inadequate. A follow-up study based on our results could monitor if the implementation of more sophisticated diagrams changes the thermodynamical results.

Future work could also implement the Polyakov loop to mimic the quark confinement [25,54-56]. It would also be interesting to perform calculations for nonzero chemical potential to explore the QCD phase diagram [24] or calculations for finite isospin [25]. The implementation of the strange quark [7,8] in an SU(3) quark-meson model and, if applicable, vector mesons [57,58] could yield a realistic model for astrophysical applications, such as for proto neutron stars or neutron star merger $[59,60]$. In $[48,61,62]$, we have already shown that the SU(3) approach in the mean field approximation yields realistic compact star scenarios. Hence, the expansion of the SU(3) quarkmeson model to finite temperatures with the vacuum term or a combined approach with quark and meson fields in the mean field approximation could indeed yield an appropriate model for a quark based equation of state for astrophysical application.

\section{ACKNOWLEDGMENTS}

The authors thank Dirk Rischke, Rainer Stiele, and Thorben Graf for discussions during the initial stage of this project. Furthermore we want to thank Konrad Tywoniuk (CERN) for helpful suggestions concerning the renormalization process.
[1] T. Toimela, Int. J. Theor. Phys. 24, 901 (1985).

[2] A. Mocsy, I. N. Mishustin, and P. J. Ellis, Phys. Rev. C 70, 015204 (2004).

[3] E. Braaten and A. Nieto, Phys. Rev. Lett. 76, 1417 (1996).

[4] E. S. Fraga and P. Romatschke, Phys. Rev. D 71, 105014 (2005).

[5] S. Gasiorowicz and D. A. Geffen, Rev. Mod. Phys. 41, 531 (1969).

[6] V. Koch, Int. J. Mod. Phys. E 06, 203 (1997).

[7] F. Karsch, E. Laermann, and A. Peikert, Nucl. Phys. B605, 579 (2001).

[8] F. Karsch, E. Laermann, A. Peikert, C. Schmidt, and S. Stickan, in Strong and Electroweak Matter. Proceedings, Meeting, SEWM 2000, Marseille, France, 2000 (World
Scientific, Singapore, 2000) pp. 180-185, DOI: 10.1142/ 9789812799913_0020.

[9] D. Zschiesche, P. Papazoglou, S. Schramm, C. Beckmann, J. Schaffner-Bielich, H. Stöcker, and W. Greiner, Springer Tracts Mod. Phys. 163, 129 (2000).

[10] D. Parganlija, P. Kovacs, G. Wolf, F. Giacosa, and D. Rischke, AIP Conf. Proc. 1520, 226 (2013).

[11] D. Parganlija, P. Kovacs, G. Wolf, F. Giacosa, and D. H. Rischke, Phys. Rev. D 87, 014011 (2013).

[12] S. Gavin, A. Goksch, and R. D. Pisarski, Phys. Rev. D 49, R3079 (1994).

[13] S. Chandrasekharan, D. Chen, N. Christ, W. Lee, R. Mawhinney, and P. Vranas, Phys. Rev. Lett. 82, 2463 (1999).

[14] D. A. Kirzhnits and A. D. Linde, Phys. Lett. B 42, 471 (1972). 
[15] R. D. Pisarski and F. Wilczek, Phys. Rev. D 29, 338 (1984).

[16] O. Kaymakcalan and J. Schechter, Phys. Rev. D 31, 1109 (1985).

[17] M. Gell-Mann and M. Levy, Nuovo Cimento 16, 705 (1960).

[18] M. Gell-Mann, Phys. Rev. Lett. 12, 155 (1964).

[19] J. B. Kogut, M. Stone, H. W. Wyld, S. Shenker, J. Shigemitsu, and D. K. Sinclair, Nucl. Phys. B225, 326 (1983).

[20] V. Koch, Phys. Lett. B 351, 29 (1995).

[21] B.-J. Schaefer and J. Wambach, Nucl. Phys. A757, 479 (2005).

[22] D. Parganlija, F. Giacosa, and D. H. Rischke, Phys. Rev. D 82, 054024 (2010).

[23] U. S. Gupta and V. K. Tiwari, Phys. Rev. D 85, 014010 (2012).

[24] T. K. Herbst, M. Mitter, J. M. Pawlowski, B.-J. Schaefer, and R. Stiele, Phys. Lett. B 731, 248 (2014).

[25] R. Stiele, E. S. Fraga, and J. Schaffner-Bielich, Phys. Lett. B 729, 72 (2014).

[26] J. T. Lenaghan and D. H. Rischke, J. Phys. G 26, 431 (2000).

[27] J. T. Lenaghan, D. H. Rischke, and J. Schaffner-Bielich, Phys. Rev. D 62, 085008 (2000).

[28] M. Grahl, E. Seel, F. Giacosa, and D. H. Rischke, Phys. Rev. D 87, 096014 (2013).

[29] E. Seel, S. Struber, F. Giacosa, and D. H. Rischke, Phys. Rev. D 86, 125010 (2012).

[30] P. Adhikari, J. O. Andersen, and P. Kneschke, Phys. Rev. D 95, 036017 (2017).

[31] Y. Hara, Y. Nambu, and J. Schechter, Phys. Rev. Lett. 16, 380 (1966).

[32] V. Bernard, A. H. Blin, B. Hiller, Y. P. Ivanov, A. A. Osipov, and U.-G. Meißner, Ann. Phys. (N.Y.) 249, 499 (1996).

[33] K. Schertler, S. Leupold, and J. Schaffner-Bielich, Phys. Rev. C 60, 025801 (1999).

[34] M. Buballa, Phys. Rep. 407, 205 (2005).

[35] V. Skokov, B. Friman, E. Nakano, K. Redlich, and B. J. Schaefer, Phys. Rev. D 82, 034029 (2010).

[36] S. Chatterjee and K. A. Mohan, Phys. Rev. D 85, 074018 (2012).

[37] R. Khan, J. O. Andersen, L. T. Kyllingstad, and M. Khan, Int. J. Mod. Phys. A 31, 1650025 (2016).
[38] D. Bailin, J. Cleymans, and M. D. Scadron, Phys. Rev. D 31, 164 (1985).

[39] J. Berges, D. U. Jungnickel, and C. Wetterich, Phys. Rev. D 59, 034010 (1999).

[40] J. M. Cornwall, R. Jackiw, and E. Tomboulis, Phys. Rev. D 10, 2428 (1974).

[41] G. Mandanici, Int. J. Mod. Phys. A 19, 3541 (2004).

[42] J. Schechter and Y. Ueda, Phys. Rev. D 3, 2874 (1971).

[43] C. Vafa and E. Witten, Nucl. Phys. B234, 173 (1984).

[44] N. A. Törnqvist, arXiv:hep-ph/9711483.

[45] A. Bochkarev and J. I. Kapusta, Phys. Rev. D 54, 4066 (1996).

[46] O. Scavenius, A. Mocsy, I. N. Mishustin, and D. H. Rischke, Phys. Rev. C 64, 045202 (2001).

[47] B.-J. Schaefer and M. Wagner, Phys. Rev. D 79, 014018 (2009).

[48] A. Zacchi, R. Stiele, and J. Schaffner-Bielich, Phys. Rev. D 92, 045022 (2015).

[49] V. K. Tiwari, Phys. Rev. D 88, 074017 (2013).

[50] A. Pilaftsis and D. Teresi, Nucl. Phys. B874, 594 (2013).

[51] N. Dupuis, Phys. Rev. B 89, 035113 (2014).

[52] H. van Hees and J. Knoll, Phys. Rev. D 65, 025010 (2001).

[53] S. Borsanyi, Z. Fodor, C. Hoelbling, S. D. Katz, S. Krieg, C. Ratti, K. K. Szabo, and Wuppertal-Budapest Collaboration, J. High Energy Phys. 09 (2010) 073.

[54] C. Sasaki, B. Friman, and K. Redlich, Phys. Rev. D 75, 074013 (2007).

[55] B.-J. Schaefer, J. M. Pawlowski, and J. Wambach, Phys. Rev. D 76, 074023 (2007).

[56] R. Stiele and J. Schaffner-Bielich, Phys. Rev. D 93, 094014 (2016).

[57] G. W. Carter, P. J. Ellis, and S. Rudaz, Nucl. Phys. A618, 317 (1997).

[58] G. W. Carter, P. J. Ellis, and S. Rudaz, Nucl. Phys. A603, 367 (1996); A608, 514(E) (1996).

[59] O. Heinimann, M. Hempel, and F.-K. Thielemann, Phys. Rev. D 94, 103008 (2016).

[60] M. Hanauske, K. Takami, L. Bovard, L. Rezzolla, J. A. Font, F. Galeazzi, and H. Stoecker, Phys. Rev. D 96, 043004 (2017).

[61] A. Zacchi, M. Hanauske, and J. Schaffner-Bielich, Phys. Rev. D 93, 065011 (2016).

[62] A. Zacchi, L. Tolos, and J. Schaffner-Bielich, Phys. Rev. D 95, 103008 (2017). 